The University of Southern Mississippi

The Aquila Digital Community

Faculty Publications

$10-7-2015$

\title{
A Generalized Force-Modified Potential Energy Surface For Mechanochemical Simulations
}

Gopinath Subramanian

University of Southern Mississippi, Gopinath.Subramanian@usm.edu

Nithin Mathew

University of Missouri

Jeff Leiding

Los Alamos National Laboratory Theoretical Division

Follow this and additional works at: https://aquila.usm.edu/fac_pubs

Part of the Biological and Chemical Physics Commons

\section{Recommended Citation}

Subramanian, G., Mathew, N., Leiding, J. (2015). A Generalized Force-Modified Potential Energy Surface For Mechanochemical Simulations. Journal of Chemical Physics, 143(13).

Available at: https://aquila.usm.edu/fac_pubs/18664

This Article is brought to you for free and open access by The Aquila Digital Community. It has been accepted for inclusion in Faculty Publications by an authorized administrator of The Aquila Digital Community. For more information, please contact Joshua.Cromwell@usm.edu. 


\title{
A Generalized Force-Modified Potential Energy Surface (G-FMPES) for Mechanochemical Simulations
}

\author{
Gopinath Subramanian, ${ }^{1}$ Nithin Mathew, ${ }^{2}$ and Jeff Leiding ${ }^{3}$ \\ ${ }^{1}$ School of Polymers and High Performance Materials, \\ University of Southern Mississippi, Hattiesburg, MS 39402* \\ ${ }^{2}$ Department of Chemistry, University of Missouri-Columbia, Columbia, MO 65211 \\ ${ }^{3}$ Theoretical Division, Los Alamos National Laboratory, Los Alamos, NM 87545
}

(Dated: September 21, 2015)

\begin{abstract}
We describe the modifications that a spatially varying external load produces on a Born-Oppenheimer potential energy surface (PES) by calculating static quantities of interest. The effects of the external loads are exemplified using electronic structure calculations (at the $\mathrm{HF} / 6-31 \mathrm{G} * *$ level) of two different molecules: ethane and hexahydro-1,3,5-trinitro-s-triazine (RDX). The calculated transition states and Hessian matrices of stationary points show that spatially varying external loads shift the stationary points and modify the curvature of the PES, thereby affecting the harmonic transition rates by altering both the energy barrier as well as the prefactor. The harmonic spectra of both molecules are blueshifted with increasing compressive "pressure." Some stationary points on the RDX-PES disappear under application of the external load, indicating the merging of an energy minimum with a saddle point.
\end{abstract}

Keywords: mechanochemistry, energy landscape, transition states, conformation, vibrational spectra

\section{INTRODUCTION}

Mechanochemistry is the use of mechanical forces to modify the potential energy surface of a system, and consequently, modify reaction pathways and rates. Recently, there has been a resurgence of interest in examining systems that have a subset of their atoms subjected to an external force. Examples include computing activation energies for defect diffusion in the vicinity of dislocations $[1,2]$, ring opening of cyclobutene [3], the design of mechanophores [4], inducing forbidden reactions by violating the Woodward-Hoffman rules [5], exploring the energy landscapes of mechanosensitive molecules [6], and mechanophore activation [7-9].

The majority of studies to date assume, for simplicity, that the external force applied on the atoms is constant, but, this need not always be the case. One example of a spatially varying force is seen in problems relevant to irradiation damage where the transition rates of defects (such as single interstitials) in metals and oxides have been demonstrated to be a function of the macroscopic stress [10-13]. In these cases, defects are embedded in a uniform stress field, but transmission of the macroscopically uniform stress to the atomic level almost inevitably results in the individual atoms experiencing a force that is a complex function of the current atomic configuration. Consequently, atoms involved in a transition will experience an external force that is a complex function of the reaction progress. Another example of a spatially varying force is seen in energetic materials such as RDX in either the presence of a macroscopic stress field [14-16], or in the vicinity of defects [17-21]. Here, as with the case of irradiation damage, the force on the atoms involved in a transition is a function of their position within the crystal. A third example is seen in molecules embedded in a non-uniform external

\footnotetext{
* Gopinath.Subramanian@usm.edu; Corresponding author
}

field (e.g., as seen in ion traps [22] where the confining external potential is quadratic) which results in a force that is non-uniform throughout the molecule, and therefore, the assumption of a constant force can be a poor approximation. A fourth example, as pointed out by a reviewer, is seen in singlemolecule force spectroscopy [23, 24] where the constant force assumption is valid only in the limit of "soft" handles through which the force is transmitted to the molecule. Therefore, a more realistic description of single-molecule pulling would also involve forces that are not constant.

The effect of any external force (either constant or spatially varying) on a subset of atoms serves to modify the potential energy surface (PES) of the system being studied, and in this article, we present a formulation for describing a Generalized Force-Modified Potential Energy Surface (G-FMPES). This formulation can be used for both constant as well as spatially varying forces, and can be viewed as a generalization of earlier work by Ong et al. [3]. This formulation also shows that spatially varying forces result in the G-FMPES having a curvature different from that of the unmodified PES: this curvature change can be particularly important for studies that explore the G-FMPES by numerical integration [6], where the tacit assumption of unchanging curvatures is made. With the formulation of the G-FMPES in place, we show that standard atomistic techniques can be used to calculate quantities of practical interest, such as transition states and vibration spectra, under the influence of a spatially varying external force. We use ethane $\left(\mathrm{C}_{2} \mathrm{H}_{6}\right)$ and hexahydro-1,3,5-trinitro-s-triazine (RDX) as our two test molecules. 


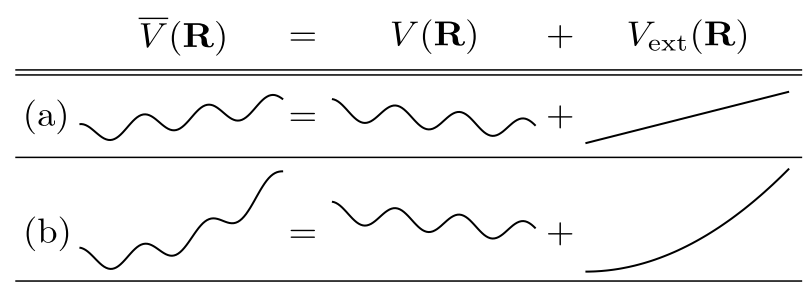

FIG. 1. A 1-dimensional generalized force-modified potential energy surface (G-FMPES), $\bar{V}(\mathbf{R})$, as the sum of an unmodified PES and an external potential. The schematic shows the results of adding two different kinds of external potentials: (a) linear, corresponding to a constant external force that preserves the curvature of the unmodified PES, and (b) quadratic, corresponding to a spatially varying force that changes the curvature of the unmodified PES.

\section{METHODS}

\section{A. The Generalized Force-Modified Potential Energy Surface (G-FMPES)}

Consider a 3-dimensional system comprised of $N$ atoms where positions of all the atoms are defined by the $3 N$ dimensional vector $\mathbf{R}$. Let the functional $V(\mathbf{R})$ describe the unmodified PES of the interaction between atoms. We assume that the PES is smooth and that third derivatives exist at all points. Thus, the force on all the atoms is given by the negative gradient of the potential energy functional as

$$
\mathbf{F}_{\text {grad }}(\mathbf{R})=-\nabla V(\mathbf{R})
$$

In mechanochemical simulations, it is necessary to apply external forces on a subset of atoms. This external force, denoted by the $3 N$-dimensional vector $\mathbf{F}_{\text {ext }}(\mathbf{R})$, is considered to be the result of an external potential, denoted by $V_{\text {ext }}(\mathbf{R})$. These last two quantities are related by the familiar expression

$$
\mathbf{F}_{\text {ext }}(\mathbf{R})=-\nabla V_{\text {ext }}(\mathbf{R})
$$

This external force, whose form is as yet unspecified, modifies the PES and results in a generalized force-modified potential energy surface (G-FMPES) given by the functional

$$
\bar{V}(\mathbf{R})=V(\mathbf{R})+V_{\text {ext }}(\mathbf{R})
$$

This addition of potential energy functionals is represented schematically for a 1-dimensional PES in figure 1 for an external potential that is (a) linear, and (b) quadratic. From this point onwards, we will adopt the convention that a variable with an overline refers to a quantity computed on a G-FMPES. In mechanochemical simulations, it is often the case that the unmodified PES and external forces are prescribed, making it necessary to calculate the numerical value of $V_{\text {ext }}(\mathbf{R})$. As long as $\mathbf{F}_{\text {ext }}(\mathbf{R})$ is a conservative field, this is achieved by choosing a suitable reference configuration, say $\mathbf{R}_{\text {ref }}$, and performing a path integral of the external force from the reference configu- ration to the desired configuration $\mathbf{R}$ as

$$
\begin{aligned}
V_{\text {ext }}(\mathbf{R})-V_{\text {ext }}\left(\mathbf{R}_{\text {ref }}\right) & =\int_{\mathbf{s}=\mathbf{R}_{\text {ref }}}^{\mathbf{s}=\mathbf{R}}-\mathbf{F}_{\text {ext }}(\mathbf{s}) \cdot \mathrm{d} \mathbf{s} \\
& =\int_{\mathbf{s}=\mathbf{R}}^{\mathbf{s}=\mathbf{R}_{\text {ref }}} \mathbf{F}_{\text {ext }}(\mathbf{s}) \cdot \mathrm{d} \mathbf{s}
\end{aligned}
$$

Note that in the second integral, removal of the negative sign results in reversal of the integration path. The actual path is unimportant because of the gradient theorem for line integrals, and therefore, a straight line is chosen as it is possibly the simplest path. In most atomistic simulations, we are interested in relative heights of points on the G-FMPES and therefore set $V_{\text {ext }}\left(\mathbf{R}_{\text {ref }}\right)=0$. Equation 3 for the G-FMPES therefore becomes

$$
\bar{V}(\mathbf{R})=V(\mathbf{R})+\int_{\mathbf{s}=\mathbf{R}}^{\mathbf{s}=\mathbf{R}_{\text {ref }}} \mathbf{F}_{\mathrm{ext}}(\mathbf{s}) \cdot \mathrm{d} \mathbf{s}
$$

and shows that the application of any external force can, in general, produce stationary points that are different from those on the unmodified PES.

As discussed in the introduction, the external force vector is not necessarily a constant. However, if we set $\mathbf{F}_{\text {ext }}$ to be a constant, as has been the case with many mechanochemical studies [3-9], equation 6 for the G-FMPES reduces to

$$
\bar{V}(\mathbf{R})=V(\mathbf{R})+\mathbf{F}_{\mathrm{ext}} \cdot\left(\mathbf{R}_{\mathrm{ref}}-\mathbf{R}\right)
$$

which is equivalent to the expression that previous researchers have employed. Thus, if we make the simplifying assumptions that previous researchers have made, our more general expression for the G-FMPES (equation 6) reduces to the more simple form employed in the past (equation 7).

\section{B. Curvature of the G-FMPES}

The local curvature of the G-FMPES is given by the Hessian matrix, which is in turn defined as the Jacobian matrix of the gradient vector of the potential energy functional. Denoting the Hessian matrix for the G-FMPES by $\overline{\mathbf{H}}(\mathbf{R})$, and applying the definition of the Hessian matrix to the definition of a G-FMPES (equation 3), we obtain

$$
\begin{aligned}
\overline{\mathbf{H}}(\mathbf{R}) & =\mathbf{J}[\nabla \bar{V}(\mathbf{R})] \\
& =\mathbf{J}\left[\nabla\left\{V(\mathbf{R})+V_{\text {ext }}(\mathbf{R})\right\}\right]
\end{aligned}
$$

The distributive property of the Jacobian and gradient operators yields an expression for the Hessian matrix on the GFMPES in terms of the Hessian matrix on the unmodified PES and the first derivatives of the external force vector as

$$
\begin{aligned}
\overline{\mathbf{H}}(\mathbf{R}) & =\mathbf{H}(\mathbf{R})-\mathbf{J}\left[\mathbf{F}_{\mathrm{ext}}(\mathbf{R})\right] \\
& =\mathbf{H}(\mathbf{R})+\mathbf{H}_{\mathrm{ext}}(\mathbf{R})
\end{aligned}
$$

where $\mathbf{H}(\mathbf{R})$ is the Hessian matrix of the unmodified PES, and 
we have designated

$$
\mathbf{H}_{\text {ext }}(\mathbf{R})=-\mathbf{J}\left[\mathbf{F}_{\text {ext }}(\mathbf{R})\right]
$$

Equations 9 and 10 show that if the external force $\mathbf{F}_{\text {ext }}$ is a constant, then $\mathbf{H}_{\mathrm{ext}}(\mathbf{R})=0$, and the Hessian of a point on the G-FMPES is the same as that of the unmodified PES, and the two surfaces have the same curvature (as shown in figure 1a). As discussed in the introduction, the external forces are not necessarily constant, and therefore, curvatures of a G-FMPES are generally expected to be different from those of the unmodified PES. The effect of varying curvatures is discussed in section II D.

\section{Pseudo-hydrostatic pressure}

As an example of a loading modality where the external force is not a constant, we use a force vector that resembles hydrostatic pressure, and we refer to this loading modality as "pseudo-hydrostatic pressure." Here, the external force applied on each atom, $\mathbf{f}_{\mathrm{ext}}^{(j)}$, is calculated as

$$
\mathbf{f}_{\mathrm{ext}}^{(j)}=P\left[\mathbf{r}^{(j)}-\mathbf{c}\right] \quad ; \quad \forall \quad j=1,2,3 \ldots N
$$

where $P$ is a user-defined "pressure" having units of kcal $\mathrm{mol}^{-1} \AA^{-2}, \mathbf{r}^{(j)}$ is the position vector of the $j^{\text {th }}$ atom, and $\mathbf{c}$ is the geometric centroid of the system. Note that the lowercase boldface symbols used here refer to 3-dimensional vectors. With this prescription, a negative value of $P$ implies compression while a positive value implies expansion. Also, both the magnitude and the direction of the external force on each atom are a function of the configuration. Consequently, the external force vector on all atoms, $\mathbf{F}_{\text {ext }}$ as in equation 6 , is a function of the current configuration and is not constant. As pointed out by a reviewer, this prescription for the application of external forces is equivalent to placing every atom of the system in the same parabolic potential centered at the geometric centroid $\mathbf{c}$. It results in a conservative force field, thereby satisfying the assumptions made in deriving equation 6 . For the interested reader, a proof that this loading modality results in a conservative force field is available in the supplementary material [25].

This prescription for mimicking hydrostatic pressure is not necessarily ideal and other prescriptions that better mimic the application of hydrostatic pressure on a system are conceivable. For example, in periodic systems this is accomplished with relative ease by simply using lattice parameters that are different from equilibrium. However, exploration of these various prescriptions is beyond the scope of this article and delegated to a future publication. These hypothetical alternate prescriptions would, however, have to satisfy the condition of producing a conservative external force field in order for equation 6 to be path independent.

\section{Transition rates on the G-FMPES}

There are two ways in which transition rates on a G-FMPES are different from those on an unmodified PES, and can be understood within the context of harmonic Transition State Theory (h-TST). Assuming that h-TST is valid on a G-FMPES, transition rates are computed as

$$
\bar{k}=\bar{k}_{0} \exp \left[-\frac{\bar{E}_{a}}{k_{B} T}\right]
$$

where $\bar{E}_{a}$ is the activation energy for a transition, $\bar{k}_{0}$ is the prefactor (related to the curvature), $k_{B}$ is the Boltzmann constant, and $T$ is the temperature.

The activation energy $\bar{E}_{a}$ is simply the difference in energies of the saddle point and the energy minimum on the G-FMPES. From equation 6 , it is easy to see that stationary points on a G-FMPES (i.e. points with $\nabla \bar{V}(\mathbf{R})=0$ ) are in general different from those on an unmodified PES, and consequently, the activation energy for a transition can be expected to be different on a G-FMPES. Calculating the activation energy requires finding the saddle point configuration, which can be achieved using any of the various saddle-point finding methods [26], and indeed, many researchers have accounted for this change $[1,10,11]$ in activation energy of a transition when computing transition rates.

However, these very studies have neglected the effects changes in curvature which affect transition rates via the prefactor $\bar{k}_{0}$. For example, studies involving point defect migration rates with which the primary author of this paper was involved $[10,11]$ have neglected curvature change effects with the justification that the most significant changes in transition rates are manifested by changes in the activation energy, which is inside the exponential of the expression for transition rates, and therefore overwhelms any effects from curvature changes that manifest themselves in the prefactor. While this assumption may be acceptable in many situations, transitions with unusual prefactors such as the transformation of voids to stacking fault tetrahedra [27] with a prefactor tens of orders of magnitude higher than typical exist, and can be expected to show counterintuitive changes in transition rates when the external force is not a constant.

The effects of curvature change on the prefactor can be understood from Vineyard's expression [28] for the prefactor:

$$
\bar{k}_{0}=\frac{\prod_{j=1}^{3 N-m} \bar{v}_{j}^{(\min )}}{\prod_{j=1}^{3 N-(m+1)} \bar{v}_{j}^{(\mathrm{sad})}}
$$

where the $3 N-m$ different $\bar{v}_{j}^{(\min )}$ are the non-zero normal mode frequencies of the energy minimum, and the $3 N-(m+1)$ different $\bar{v}_{j}^{\text {(sad) }}$ are the positive and non-zero normal mode frequencies of the saddle point for the transition. The subtraction of $m$ in the numerator (energy minimum) accounts for 
the $m$ rigid-body motion degrees of freedom, and the subtraction of $(m+1)$ in the denominator (saddle point) accounts for the rigid-body motion degrees of freedom plus 1 imaginary frequency for a first-order saddle point. For systems that have translational and rotational symmetry, $m=6$. Application of external forces can break the symmetry, resulting in $m \leq 6$. These normal mode frequencies are in turn calculated by diagonalizing the appropriate Hessian matrix $\overline{\mathbf{H}}(\mathbf{R})=\mathbf{H}(\mathbf{R})+\mathbf{H}_{\text {ext }}(\mathbf{R})$.

For a constant external force resulting in $\mathbf{H}_{\text {ext }}(\mathbf{R})=0$, the prefactor $\bar{k}_{0}$ is calculated using the curvature of the unmodified PES at the locations of the new stationary points. However, as stated previously, there can be situations where the curvature change has to be accounted for.

\section{APPLICATIONS}

With the above understanding of how to compute energies, forces, and curvatures on a G-FMPES, standard atomistic techniques can now be used in a straightforward manner to compute quantities of practical interest. In the remainder of this article, we will demonstrate the effect of pseudohydrostatic pressure on transition states and harmonic transition rates for conformational changes, and harmonic vibrational spectra of two selected molecules, ethane $\left(\mathrm{C}_{2} \mathrm{H}_{6}\right)$ and hexahydro-1,3,5-trinitro-s-triazine (RDX).

The Hartree-Fock (HF) method [29-31] with the 6-31G** basis set [32], as implemented in the AIMS-MOLPRO code [33], was used for computing energies and gradients on the unmodified PES. While this combination of method and basis set is not necessarily ideal for purposes of accuracy, it serves as a computationally inexpensive means of illustrating the main point of this paper: the calculation of static quantities on a G-FMPES and their comparison with corresponding quantities on an unmodified PES. Custom Python routines were used in conjunction with MOLPRO to explore the G-FMPES. Transition states were calculated using the Nudged Elastic Band (NEB) with two climbing images [34] and the improved tangent estimate [35]. A more detailed description of our implementation is available in the supplementary material[25]. Structure optimizations (for finding energy minima as well as transition states) were performed using the Fast Inertial Relaxation Engine (FIRE) [36]. The integral in equation 6 was computed using the trapezoidal rule over a grid with resolution $5 \times 10^{-3} \AA$ along a straight line between $\mathbf{R}$ and $\mathbf{R}_{\text {ref }}$. The Hessians of all stationary points on the G-FMPES had 6 eigenvalues that were numerically zero and corresponded to translation and rigid body rotation. The Hessians of all saddle points were verified as having only one negative eigenvalue, thereby ensuring that they were true first-order saddles.

We first examined the rotational barrier in ethane, which is a $120^{\circ}$ rotation of a $\mathrm{CH}_{3}$ group about the $\mathrm{C}-\mathrm{C}$ bond of an ethane molecule, as shown in figure 2 where the cyan atoms are carbon and the white atoms are hydrogen. The rotational barrier in ethane is a prototypical example for developing an understanding of steric hindrance. It was first discovered by Kemp and Pitzer [37] who showed that an internal rotational

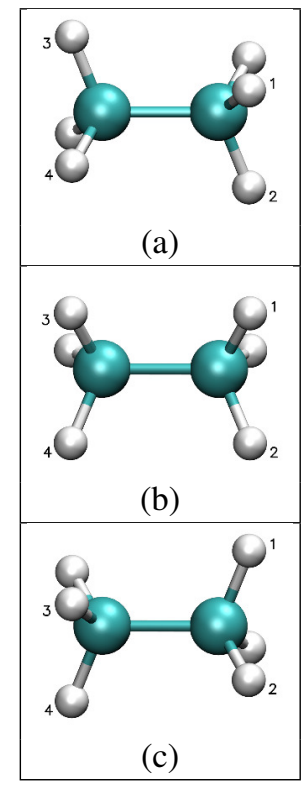

FIG. 2. Rotation of a $\mathrm{CH}_{3}$ group by $120^{\circ}$ about the $\mathrm{C}-\mathrm{C}$ bond in ethane. (a) Start state - often referred to as the staggered conformation (b) Saddle point or eclipsed conformation (c) End state - also a staggered conformation.

barrier of $2.8 \mathrm{kcal} \mathrm{mol}^{-1}$ had to be present in order to obtain thermodynamic quantities in agreement with experiment. This classic problem remains the topic of theoretical analyses as the origins of the barrier are still being probed [38, 39], and to our knowledge, the effect of pressure on this barrier has not been investigated.

For this transition, we chose the staggered conformation at $P=0$ to be the reference configuration $\mathbf{R}_{\text {ref }}$ with $V_{\text {ext }}\left(\mathbf{R}_{\text {ref }}\right)=$ 0 . Figure 3 a shows the energy along the reaction coordinate for this transition at various pressures, and all energies are relative to the reference configuration. For the transition with no external pressure, we obtain an energy barrier of $3.02 \mathrm{kcal}$ $\mathrm{mol}^{-1}$ which is within $8 \%$ of the experimental value of 2.8 $\mathrm{kcal} \mathrm{mol}^{-1}[37,40]$. With increasing compression (or decreasing values of $P$ ), we see that the energy of the saddle point progressively increases while the energy of the staggered conformation decreases and thus leads to a net increase in activation energy as a function of increasing compression. There is a concomitant increase in the prefactor with increasing compression (see figure 3), but this increase is overshadowed by the increase in activation energy, and consequently, there is a net decrease in the transition rate as a function of increasing compression. We speculate that under the influence of compressive pressure, the molecule adopts a more compact conformation which leads to atoms interacting more strongly with each other. This leads to more pronounced steric effects and a net decrease in transition rates.

The wavenumbers of the staggered conformation of ethane computed by diagonalizing the Hessian are given in table I in units of inverse centimeters. The wavenumbers at zero pressure are in good agreement with the values available in the literature for calculations at the $\mathrm{HF} / 6-31 \mathrm{G}^{* *}$ level [41]. We see 


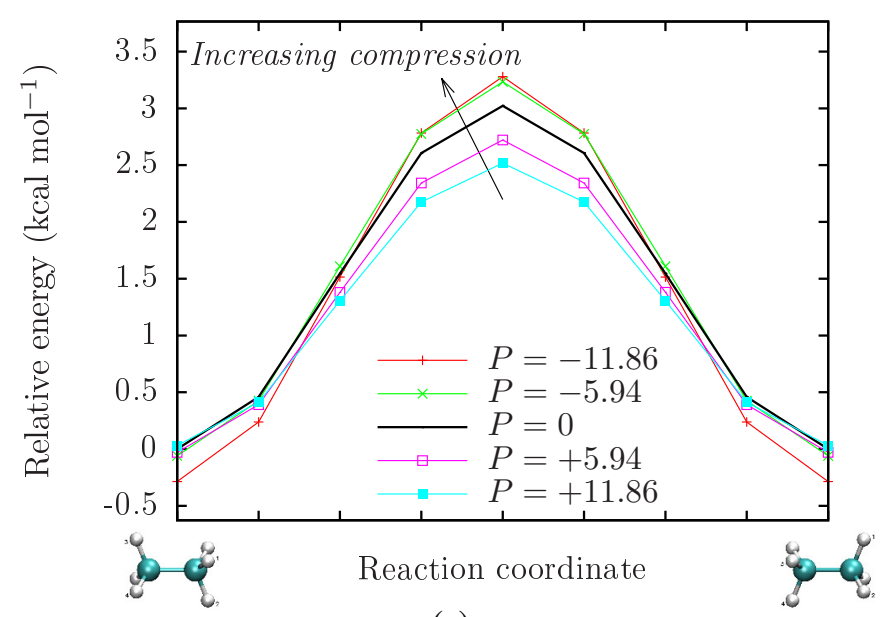

(a)

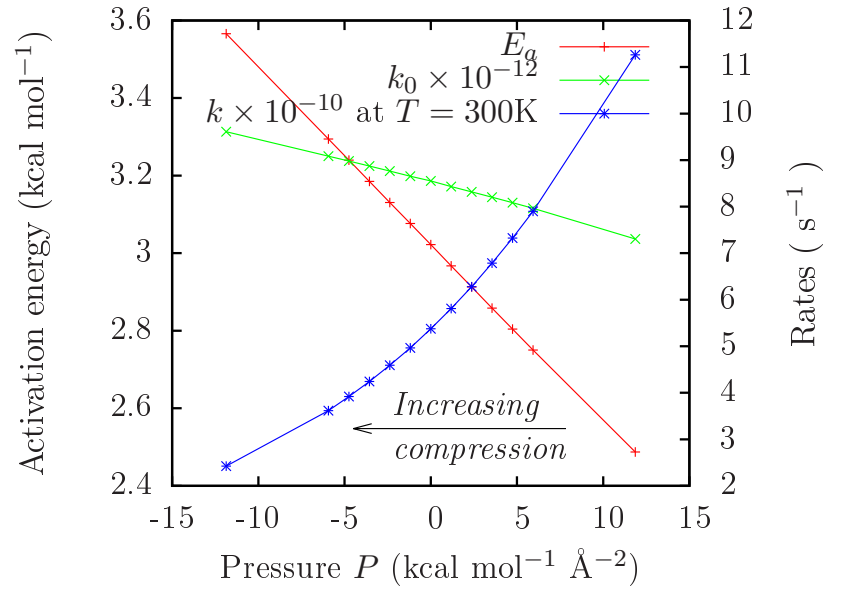

(b)

FIG. 3. For the rotational transition of ethane, (a) energy along the reaction coordinate as a function of pressure for the values of $P$ (with units of $\mathrm{kcal} \mathrm{mol}^{-1} \AA^{-2}$ ) indicated in the legend, and (b) activation energies, prefactors, and transition rates as a function of pressure. All energies are relative to that of the staggered configuration at zero pressure. On both figures, an arrow indicates the direction of increasing compression.

TABLE I. Calculated wavenumbers (in $\mathrm{cm}^{-1}$ ) of the staggered conformation of ethane for the lowest, zero, and highest values of pseudo-hydrostatic pressure (in $\mathrm{kcal} \mathrm{mol}^{-1} \AA^{-2}$ ) studied. The wavenumbers of the rigid body modes of deformation are not reported.

\begin{tabular}{ccc}
\hline$P=-11.86$ & $P=0$ & $P=11.86$ \\
\hline 382.80 & 327.65 & 264.69 \\
897.95 & 885.11 & 846.16 \\
897.95 & 885.16 & 876.95 \\
1208.96 & 1059.31 & 876.96 \\
1366.90 & 1329.27 & 1281.82 \\
1366.91 & 1329.31 & 1281.82 \\
1510.56 & 1531.93 & 1538.96 \\
1574.90 & 1564.79 & 1546.27 \\
1648.04 & 1627.73 & 1601.37 \\
1648.12 & 1627.81 & 1601.43 \\
1651.56 & 1632.78 & 1612.73 \\
1651.64 & 1632.86 & 1612.79 \\
3344.70 & 3180.24 & 2992.31 \\
3351.69 & 3185.88 & 2995.89 \\
3423.52 & 3234.15 & 3020.84 \\
3423.60 & 3234.22 & 3020.91 \\
3445.54 & 3259.17 & 3047.84 \\
3445.61 & 3259.23 & 3047.90 \\
\hline
\end{tabular}

that compressive pressures shift the vibrational spectrum to higher wavenumbers (blueshift) indicating that the modes become stiffer, while expansive pressures have the opposite effect, i.e., redshift the spectrum. This behavior is qualitatively consistent with the experimental behavior seen by Kurnosov et al. [42] who saw that the Raman spectra of both liquid and solid ethane shifted to higher wavenumbers with increasingly compressive pressure over a range of up to $6900 \mathrm{MPa}$. However, the amount of shift they saw is significantly lower (approximately a factor 10) than the shifts we see in our simulations, indicating that the simulated pseudo-hydrostatic pressures on ethane are likely higher than the experimental values.

The second molecule examined was hexahydro-1,3,5trinitro-s-triazine (RDX), which forms an energetic molecular crystal and is used in many explosive formulations. Five polymorphic forms of RDX, namely $\alpha$ [45], $\beta$ [44, 46, 47], $\gamma$ [48, 49], $\delta$ [50], and $\varepsilon$ [51] have been discovered, with the $\alpha$ polymorph being stable at room temperature and atmospheric pressure. RDX has a chemical formula $\mathrm{C}_{3} \mathrm{H}_{6} \mathrm{~N}_{6} \mathrm{O}_{6}$, and is shown in figure 4 . The red atoms are oxygen and are part of the nitro $\left(\mathrm{NO}_{2}\right)$ groups, blue atoms are nitrogen, and cyan atoms are carbon. The ring formed by alternating $\mathrm{C}$ and $\mathrm{N}$ atoms is the triazine ring, and the nitro groups are on the side. The conformation of an RDX molecule is determined by the shape of the triazine ring (Boat, Chair, or Twist, denoted by B, C, or T, respectively) and the position of the nitro side groups with respect to the triazine ring as determined by the angle between the N-N bond and the plane of the C-N-C triple (axial, intermediate, or equatorial, denoted by a, i, or e respectively). Electronic structure calculations [43, 52, 53] have predicted the existence of the Caaa (figure 4a), Caae, Caee, and Ceee (figure 4c) conformers in the gas-phase, along with other conformers having the triazine ring in a Boat or Twist form. The conformational state of an RDX molecule in the solid-phase depends on the type of the polymorph, state of stress, and presence of defects. All molecules in the orthorhombic unit cell of $\alpha$-RDX exist in the Caae conformation, whereas all molecules in the $\beta$ and $\varepsilon$ polymorphs are in the Caaa conformation $[49,54]$. Of the eight molecules in the $\gamma$-RDX unit-cell, four exist in the Caai conformation and the rest in the Caae conformation [15]. Conformational transitions in $\alpha$-RDX crystals have been predicted to occur due to the passage of shock waves [16], during the $\alpha-\gamma$ phase transition [15], under uniaxial strain [14], on the surface of 
TABLE II. Calculated wavenumbers (in $\mathrm{cm}^{-1}$ ) of the two conformers of RDX for the lowest, zero, and highest values of pseudo-hydrostatic pressure (in kcal mol${ }^{-1} \AA^{-2}$ ) studied. The wavenumbers of the rigid body modes of deformation are not reported. For comparison, wavenumbers obtained by Rice and Chabalowski [43] for the Caaa conformer using a B3LYP functional, as well as experimental data from the vapor phase obtained by Karpowicz and Brill [44] are shown.

\begin{tabular}{|c|c|c|c|c|c|c|c|}
\hline \multirow{2}{*}{$\begin{array}{c}\text { DFT, } \\
\text { Ref. [43] }\end{array}$} & \multirow{2}{*}{$\begin{array}{c}\text { Experimental } \\
\text { Ref. [44] }\end{array}$} & \multicolumn{3}{|c|}{ This work, Caaa } & \multicolumn{3}{|c|}{ This work, Ceee } \\
\hline & & $P=-1.78$ & $P=0$ & $P=0.59$ & $P=-1.78$ & $P=0$ & $P=0.59$ \\
\hline 31 & & 43.16 & 35.64 & 17.97 & 54.07 & 54.67 & 57.61 \\
\hline 37 & & 46.87 & 35.85 & 18.98 & 54.24 & 54.91 & 57.72 \\
\hline 63 & & 46.92 & 66.61 & 52.48 & 58.41 & 69.43 & 74.24 \\
\hline 67 & & 124.51 & 77.24 & 80.61 & 83.42 & 89.03 & 90.61 \\
\hline 100 & & 147.74 & 109.04 & 100.29 & 117.10 & 122.78 & 125.56 \\
\hline 102 & & 148.16 & 109.20 & 100.41 & 117.17 & 122.88 & 125.60 \\
\hline 220 & & 252.57 & 242.29 & 226.39 & 190.72 & 187.66 & 188.00 \\
\hline 221 & & 252.89 & 243.26 & 227.50 & 190.85 & 187.96 & 188.13 \\
\hline 301 & & 328.25 & 342.44 & 353.29 & 258.64 & 242.31 & 237.75 \\
\hline 363 & & 433.26 & 408.85 & 392.75 & 258.82 & 242.62 & 237.89 \\
\hline 365 & & 433.84 & 409.58 & 393.09 & 354.65 & 323.37 & 312.62 \\
\hline 409 & & 490.40 & 465.49 & 442.88 & 392.41 & 378.23 & 373.04 \\
\hline 413 & & 490.50 & 465.54 & 442.96 & 413.76 & 402.22 & 398.37 \\
\hline 442 & & 542.98 & 508.24 & 480.28 & 413.81 & 402.58 & 398.50 \\
\hline 458 & & 581.23 & 534.35 & 500.34 & 413.87 & 402.70 & 398.55 \\
\hline 590 & & 665.53 & 665.83 & 666.88 & 662.87 & 653.23 & 649.50 \\
\hline 590 & & 678.61 & 669.92 & 667.07 & 662.91 & 653.35 & 649.54 \\
\hline 593 & & 678.62 & 670.09 & 670.71 & 763.07 & 748.61 & 744.02 \\
\hline 660 & & 743.83 & 736.41 & 736.48 & 804.80 & 791.93 & 787.26 \\
\hline 661 & 782 & 744.39 & 737.15 & 736.95 & 804.85 & 791.98 & 787.28 \\
\hline 749 & 845 & 838.31 & 847.56 & 854.82 & 888.82 & 888.90 & 888.52 \\
\hline 753 & 880 & 893.85 & 889.89 & 887.55 & 905.48 & 900.70 & 899.43 \\
\hline 754 & & 894.12 & 890.18 & 887.70 & 905.54 & 900.76 & 899.45 \\
\hline 782 & 910 & 927.25 & 911.05 & 900.68 & 926.74 & 919.71 & 917.42 \\
\hline 863 & & 977.35 & 965.23 & 961.49 & 1025.01 & 1000.65 & 993.22 \\
\hline 864 & & 977.39 & 965.30 & 961.58 & 1025.04 & 1000.66 & 993.23 \\
\hline 887 & & 994.59 & 972.64 & 964.37 & 1039.98 & 1007.40 & 997.06 \\
\hline 906 & 1014 & 1054.98 & 1048.14 & 1049.12 & 1132.72 & 1121.19 & 1118.04 \\
\hline 907 & & 1055.04 & 1048.31 & 1049.38 & 1132.73 & 1121.31 & 1118.09 \\
\hline 935 & & 1103.13 & 1096.06 & 1096.06 & 1158.32 & 1137.97 & 1131.37 \\
\hline 1005 & & 1183.59 & 1177.26 & 1181.35 & 1245.16 & 1222.61 & 1214.21 \\
\hline 1005 & 1218 & 1183.95 & 1177.81 & 1181.77 & 1245.22 & 1222.61 & 1214.22 \\
\hline 1141 & 1268 & 1299.40 & 1289.72 & 1291.61 & 1335.81 & 1319.68 & 1313.45 \\
\hline 1242 & 1319 & 1414.78 & 1400.56 & 1399.42 & 1425.35 & 1403.41 & 1393.44 \\
\hline 1250 & 1374 & 1415.18 & 1401.04 & 1399.72 & 1438.70 & 1407.28 & 1397.35 \\
\hline 1252 & & 1421.61 & 1414.65 & 1403.04 & 1464.11 & 1439.33 & 1430.03 \\
\hline 1275 & 1420 & 1436.76 & 1418.06 & 1421.94 & 1464.18 & 1439.46 & 1430.07 \\
\hline 1292 & 1444 & 1494.98 & 1494.98 & 1497.76 & 1476.04 & 1458.18 & 1451.63 \\
\hline 1294 & & 1520.75 & 1511.62 & 1505.00 & 1476.08 & 1458.33 & 1451.70 \\
\hline 1345 & & 1520.77 & 1511.83 & 1505.27 & 1525.41 & 1518.57 & 1516.90 \\
\hline 1363 & & 1541.13 & 1531.23 & 1528.75 & 1558.34 & 1537.31 & 1530.36 \\
\hline 1381 & & 1541.77 & 1531.81 & 1528.99 & 1558.37 & 1537.38 & 1530.43 \\
\hline 1384 & 1584 & 1585.01 & 1578.37 & 1577.65 & 1636.15 & 1612.26 & 1603.91 \\
\hline 1402 & & 1585.06 & 1578.44 & 1577.72 & 1636.37 & 1623.96 & 1619.76 \\
\hline 1403 & & 1598.31 & 1589.11 & 1585.93 & 1636.42 & 1624.01 & 1619.80 \\
\hline 1464 & & 1619.50 & 1616.34 & 1618.19 & 1657.76 & 1654.11 & 1653.79 \\
\hline 1466 & & 1619.53 & 1616.43 & 1618.31 & 1657.80 & 1654.11 & 1653.79 \\
\hline 1482 & & 1665.26 & 1655.60 & 1653.42 & 1670.68 & 1668.21 & 1667.74 \\
\hline 1627 & & 1854.72 & 1845.01 & 1839.91 & 1864.45 & 1848.85 & 1843.95 \\
\hline 1658 & & 1914.64 & 1890.13 & 1877.62 & 1889.74 & 1873.60 & 1868.46 \\
\hline 1658 & & 1914.99 & 1890.69 & 1878.07 & 1889.96 & 1873.61 & 1868.48 \\
\hline 3064 & 3065 & 3311.08 & 3266.08 & 3245.22 & 3188.21 & 3172.70 & 3167.49 \\
\hline 3064 & & 3311.42 & 3266.52 & 3245.39 & 3188.29 & 3172.81 & 3167.52 \\
\hline 3070 & & 3323.14 & 3276.76 & 3254.68 & 3196.56 & 3179.52 & 3173.78 \\
\hline 3192 & & 3476.09 & 3434.69 & 3419.39 & 3484.77 & 3431.28 & 3412.98 \\
\hline 3192 & & 3476.20 & 3434.73 & 3419.44 & 3484.82 & 3431.33 & 3413.01 \\
\hline 3194 & & 3477.51 & 3436.30 & 3421.24 & 3487.38 & 3433.89 & 3415.59 \\
\hline
\end{tabular}

voids [17], and inside stacking faults [18, 19, 55] and dislocation cores [20]. Molecular dynamics simulations using a nonreactive and fully-flexible force field have shown that conformational transitions play an important role in the stability of high-pressure phases [15], plastic deformation mechanisms of RDX [19, 55-57], and contribute to energy concentration in strained $\alpha$-RDX crystals [14].

We studied the Caaa $\leftrightarrow$ Ceee transition (shown in figure 4) of an isolated RDX molecule because it has the highest ac- tivation energy of all the transitions between conformers for an RDX molecule with the Chair shaped triazine ring. The pseudo-hydrostatic pressures applied in this test case are particularly relevant because the deviatoric part of the stress tensor vanishes for the gas phase and the pressure $(-\operatorname{tr}(\sigma) / 3)$ becomes the critical term at high stresses and temperatures under which decomposition of shocked explosives takes place.

For this transition, we chose the Caaa conformer at $P=0$ to be the reference configuration $\mathbf{R}_{\text {ref }}$ with $V_{\text {ext }}\left(\mathbf{R}_{\text {ref }}\right)=0$. In- 


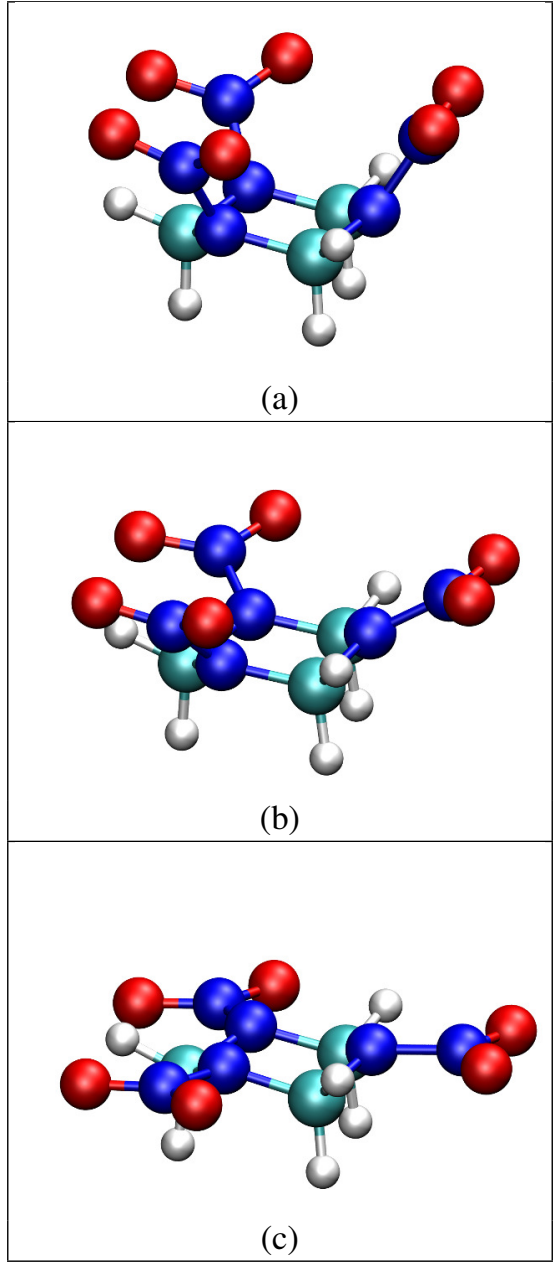

FIG. 4. Transition of an RDX molecule $\left(\mathrm{C}_{3} \mathrm{H}_{6} \mathrm{~N}_{6} \mathrm{O}_{6}\right)$ from the Caaa conformer to Ceee. (a) Caaa conformer, (b) saddle point, and (c) Ceee conformer. The red atoms are oxygen, and are part of the nitro $\left(\mathrm{NO}_{2}\right)$ groups, blue atoms are nitrogen, and cyan atoms are carbon. The ring formed by alternating $\mathrm{C}$ and $\mathrm{N}$ atoms is the triazine ring.

creasingly compressive pressures were seen to increase the energy barrier for the forward (Caaa $\rightarrow$ Ceee) transition while decreasing the barrier for the reverse (Caaa $\leftarrow$ Ceee) transition with a concomitant increase (or decrease) in the net rates (see figure 5). For pseudo-hydrostatic pressures with $P \leq-2.37$ kcal $\mathrm{mol}^{-1} \AA^{-2}$, i.e. high compressions, we observed that the Ceee conformer was no longer stable, and spontaneously transitioned into the Caaa conformer. Conversely, for pseudohydrostatic pressures with $P \geq 1.78 \mathrm{kcal} \mathrm{mol}^{-1} \AA^{-2}$, the Caaa conformer was no longer stable and spontaneously transformed into the Ceee conformer. This type of mechanism where external stresses cause existing stationary points on the energy landscape to vanish has been observed before in the case of zirconium interstitials [10], and indicates that an energy minimum merges with a saddle point (referred to as a "catastrophe" by earlier studies [58]). The opposite scenario, where external stresses introduce new stationary points into the energy landscape, was not observed for the range of pressures studied in this article but is a very real possibility for other more complex systems.

As with the case of ethane, we speculate that higher compressions cause the RDX molecule to adopt more compact conformations leading to enhancements of steric effects. This observation agrees well with the fact that the Caaa conformer is present in the high-pressure polymorphs ( $\beta$ and $\varepsilon$ ) of RDX $[49,54]$. For the forward transition (Caaa $\rightarrow$ Ceee), we see that the transition rate decreases with increasing compression. However, the backward transition (Caaa $\leftarrow$ Ceee) rate increases with increasing compression, and this is thought to be because the Ceee conformer is a higher-energy conformer where steric effects are already pronounced (as evidenced by it being unstable at high compressions) and the extra compression serves to make transitions out of this high-energy conformer more accessible.

Table II shows the calculated wavenumbers with units of $\mathrm{cm}^{-1}$ of the two conformations for the lowest, zero, and highest values of pseudo-hydrostatic pressure used in this study. Again, as with the case of ethane, the harmonic vibrational spectrum of RDX is seen to shift to higher wave numbers with increasing compression indicating stiffer modes. This behavior is consistent with previous independent experimental studies by Dreger and Gupta [59], Zheng et al. [60], and Ciezak et al. [50], as well as molecular dynamics studies [61]. However, the softer modes of Ceee, from $54-122 \mathrm{~cm}^{-1}$ behave in the opposite manner. Also shown in table II are the wavenumbers obtained from DFT studies by Rice and Chabalowski [43] (at the B3LYP/6-31G** level), as well as experimental data from the vapor phase [44]. We found good agreement between our harmonic frequencies and those of experiment. There are significant differences between our frequencies and those of Rice and Chabalowski [43], but this is can be attributed to the differences in electronic structure theory techniques employed. At this point, we remind the reader that the thrust of this article is not accurate computation of spectra, but examination of the effect of pressure on atomistically calculated quantities. Therefore, quantitative comparison between experimental data and simulations is deferred until after we gain a better understanding of the relationship between pseudo-hydrostatic pressure and its thermodynamic counterpart.

\section{CONCLUSIONS}

We have outlined a formulation for computing energies on a generalized force-modified potential energy surface (GFMPES), and demonstrated that with this formulation, calculation of atomistic quantities (including transition states) in the presence of a spatially varying force is easily accomplished using standard atomistic techniques. We have demonstrated the formulation using two simple molecules, namely ethane and RDX, and by calculating a variety of atomistic properties. For ethane, we examined the rotational barrier (a classic problem), and for RDX, we examined the Caaa $\leftrightarrow$ Ceee transition of RDX. We also calculated the 0K Hessian matrix of stationary points, and showed that for the pseudohydrostatic loading mode used in this study, the curvature of the G-FMPES is indeed different from that of the unmodified 

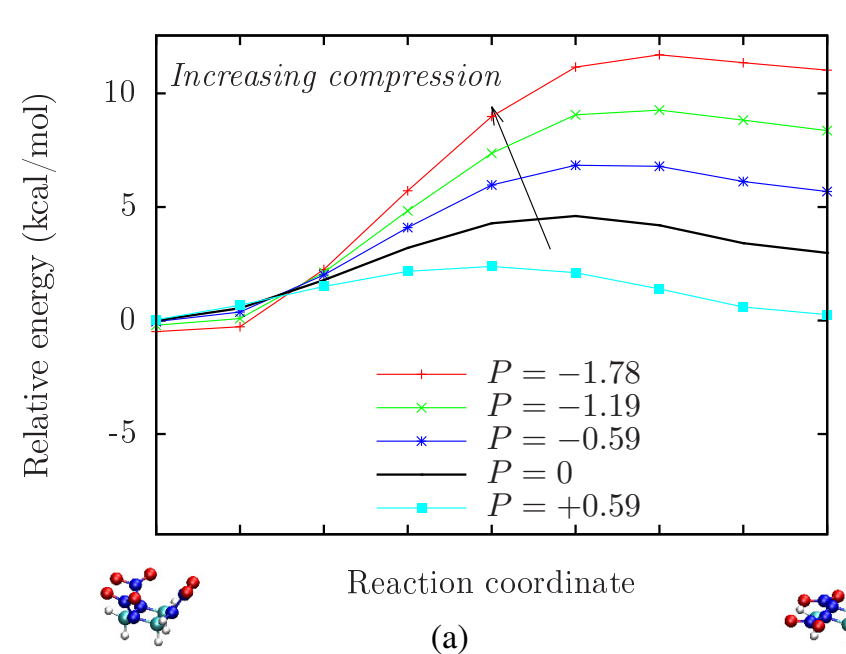

Reaction coordinate

(a)

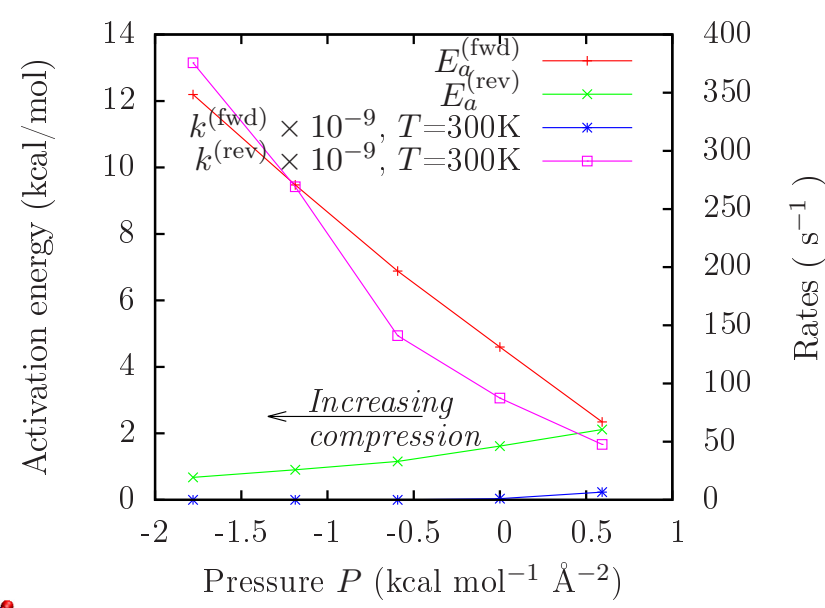

(b)

FIG. 5. For the Caaa to Ceee transition of RDX, (a) energy along the reaction coordinate as a function of pressure for the values of $P$ (with units of kcal mol ${ }^{-1} \AA^{-2}$ ) indicated in the legend, and (b) activation energies, prefactors, and transition rates as a function of pressure. Here, the forward transition is the Caaa $\rightarrow$ Ceee transition. All energies are relative to that of the Caaa conformation at zero pressure. On both figures, an arrow indicates the direction of increasing compression.

PES, and that it has an effect on the curvature and harmonic transition rates. We also see instances where the addition of an external force causes an RDX conformer to become unstable, indicating merging of an energy minimum with a saddle point. We speculate that increasing compressive pressures enhance steric effects by forcing molecules into more compact conformations. For ethane, this leads to a net increase in the rotational barrier resulting in a lower transition rate. For RDX, the activation energy for the Caaa $\rightarrow$ Ceee transition also increases. However for the backward Caaa $\leftarrow$ Ceee transition, since the Ceee conformation is already a high energy state, enhanced steric effects make transitions out of this conformer more accessible resulting in a lowering of the energy barrier. For both molecules, increasing compressive pressures shifted the vibrational spectra towards higher wavenumbers, indicating that the vibrational modes become stiffer. This behavior is qualitatively consistent with independent experiments, as well as molecular dynamics studies. With the formulation of the G-FMPES in place, it is now possible to compute dynamical quantities using standard MD techniques to incorporate the effects of spatially varying external forces on finite temperature calculations.

While the data presented in this article indicate that our formulation is valid, it is important to restate our assumptions and to point out potential avenues that might require further investigation. First and foremost, the formulation assumes that the force field of the G-FMPES is conservative. This assumption is necessary for the integral in equation 6 to be path independent. Secondly, a real possibility is the introduction of new minima into the G-FMPES. While we did not observe any intermediate minima along the transition path for the systems studied in this article, it might happen in other more complex systems. Finally, while our prescription for pseudohydrostatic pressure is reminiscent of mechanical pressure, the relationship between the two requires investigation.

\section{ACKNOWLEDGMENTS}

G.S. acknowledges startup funds from the College of Science and Technology at the University of Southern Mississippi, and thanks Art Voter, Blas Uberuaga, and Danny Perez for invaluable advice on the topic of potential energy surfaces. This paper has been designated LA-UR \# 15-23906
[1] C. Varvenne, F. Bruneval, M.-C. Marinica, and E. Clouet, Phys. Rev. B 88, 134102 (2013), URL http://link.aps.org/ doi/10.1103/PhysRevB. 88.134102.

[2] E. Clouet, S. Garruchet, H. Nguyen, M. Perez, and C. S. Becquart, Acta Materialia 56, 3450 (2008), ISSN 13596454, URL http://www.sciencedirect.com/science/ article/pii/S1359645408002218.

[3] M. T. Ong, J. Leiding, H. Tao, A. M. Virshup, and T. J. Martnez, Journal of the American Chemical Society 131, 6377 (2009), pMID: 19378993, http://dx.doi.org/10.1021/ja8095834, URL http://dx.doi.org/10.1021/ja8095834.
[4] S. S. M. Konda, J. N. Brantley, B. T. Varghese, K. M. Wiggins, C. W. Bielawski, and D. E. Makarov, Journal of the American Chemical Society 135, 12722 (2013), pMID: 23905836, http://dx.doi.org/10.1021/ja4051108, URL http://dx. doi . org/10.1021/ja4051108.

[5] J. Wang, T. B. Kouznetsova, Z. Niu, M. T. Ong, H. M. Klukovich, A. L. Rheingold, T. J. Martinez, and S. L. Craig, Nat Chem 7, 323 (2015), URL http://dx.doi.org/10.1038/ nchem. 2185.

[6] S. Sriharsha, S. M. Avdoshenko, and D. E. Makarov, The Journal of Chemical Physics 140, 104114+ (2014), ISSN 0021- 
9606, URL http://dx. doi .org/10.1063/1.4867500.

[7] M. N. Silberstein, K. Min, L. D. Cremar, C. M. Degen, T. J. Martinez, N. R. Aluru, S. R. White, and N. R. Sottos, Journal of Applied Physics 114, 023504+ (2013), ISSN 0021-8979, URL http://dx.doi.org/10.1063/1.4812581.

[8] M. N. Silberstein, L. D. Cremar, B. A. Beiermann, S. B. Kramer, T. J. Martinez, S. R. White, and N. R. Sottos, Journal of the Mechanics and Physics of Solids 63, 141 (2014), ISSN 0022-5096, URL http://www.sciencedirect.com/ science/article/pii/S0022509613001890.

[9] A. Bailey and N. J. Mosey, The Journal of Chemical Physics 136, 044102 (2012), URL http://scitation.aip.org/ content/aip/journal/jcp/136/4/10.1063/1.3678010.

[10] G. Subramanian, D. Perez, B. P. Uberuaga, C. N. Tomé, and A. F. Voter, Physical Review B 87, 144107+ (2013), URL http://dx.doi.org/10.1103/physrevb.87.144107.

[11] A. Goyal, S. R. Phillpot, G. Subramanian, D. A. Andersson, C. R. Stanek, and B. P. Uberuaga, Phys. Rev. B 91, 094103 (2015), URL http://link.aps.org/doi/10. 1103/PhysRevB.91.094103.

[12] G. Vérité, C. Domain, C.-C. Fu, P. Gasca, A. Legris, and F. Willaime, Phys. Rev. B 87, 134108 (2013), URL http: //link.aps.org/doi/10.1103/PhysRevB.87.134108.

[13] T. Garnier, V. R. Manga, P. Bellon, and D. R. Trinkle, Phys. Rev. B 90, 024306 (2014), URL http://link.aps.org/ doi/10.1103/PhysRevB.90.024306.

[14] N. Mathew and R. C. Picu, The Journal of Chemical Physics 135, 024510 (2011), URL http://scitation.aip.org/ content/aip/journal/jcp/135/2/10.1063/1.3609769.

[15] L. B. Munday, P. W. Chung, B. M. Rice, and S. D. Solares, The Journal of Physical Chemistry B 115, 4378 (2011), pMID: 21434619, http://dx.doi.org/10.1021/jp112042a, URL http: //dx.doi.org/10.1021/jp112042a.

[16] M. J. Cawkwell, T. D. Sewell, L. Zheng, and D. L. Thompson, Phys. Rev. B 78, 014107 (2008), ISSN 1098-0121, URL http: //link.aps.org/doi/10.1103/PhysRevB.78.014107.

[17] S. Boyd, J. S. Murray, and P. Politzer, J. Chem. Phys. 131, 204903 (2009), ISSN 1089-7690, URL http://www.ncbi. nlm.nih.gov/pubmed/19947705.

[18] K. J. Ramos, D. E. Hooks, T. D. Sewell, and M. J. Cawkwell, J. Appl. Phys. 108, 066105 (2010), ISSN 00218979, URL http://link . aip.org/link/JAPIAU/v108/i6/p066105/ s1 $\backslash \&$ Agg $=$ doi

[19] L. B. Munday, S. D. Solares, and P. W. Chung, Philos. Mag. 92, 37 (2012), URL http: //www. tandfonline.com/doi/abs/ 10.1080/14786435.2012.685191.

[20] N. Mathew, C. R. Picu, and P. W. Chung, J. Phys. Chem. A 117, 5326 (2013), ISSN 1520-5215, URL http: //www .ncbi .nlm. nih.gov/pubmed/23734970.

[21] A. Pal and R. C. Picu, The Journal of Chemical Physics 140, 044512 (2014), URL http://scitation.aip.org/ content/aip/journal/jcp/140/4/10.1063/1.4862997.

[22] D. E. Austin, Y. Peng, B. J. Hansen, I. W. Miller, A. L. Rockwood, A. R. Hawkins, and S. E. Tolley, Journal of the American Society for Mass Spectrometry 19, 1435 (2008), ISSN 1044-0305, URL http://www.sciencedirect.com/ science/article/pii/S1044030508002316.

[23] O. K. Dudko, G. Hummer, and A. Szabo, Proceedings of the National Academy of Sciences 105, 15755 (2008), http://www.pnas.org/content/105/41/15755.full.pdf, URL http://www.pnas.org/content/105/41/15755. abstract.

[24] K. C. Neuman and A. Nagy, Nat Meth 5, 491 (2008), ISSN 1548-7091, URL http://dx.doi.org/10.1038/nmeth.
1218.

[25] See supplementary material at [URL TO BE PROVIDED BY AIP] for proof of the conservative nature of the external force field, and a description of the NEB implementation on the GFMPES.

[26] G. Henkelman, G. Jóhannesson, and H. Jónsson, in Theoretical Methods in Condensed Phase Chemistry, edited by S. Schwartz (Springer Netherlands, 2002), vol. 5 of Progress in Theoretical Chemistry and Physics, pp. 269-302, ISBN 978-0-7923-66874, URL http://dx.doi .org/10.1007/0-306-46949-9_ 10.

[27] B. P. Uberuaga, R. G. Hoagland, A. F. Voter, and S. M. Valone, Phys. Rev. Lett. 99, 135501 (2007), URL http://link . aps. org/doi/10.1103/PhysRevLett.99.135501.

[28] G. H. Vineyard, Journal of Physics and Chemistry of Solids 3, 121 (1957), ISSN 0022-3697, URL http://www.sciencedirect.com/science/article/ pii/0022369757900598.

[29] D. R. Hartree, Mathematical Proceedings of the Cambridge Philosophical Society 24, 89 (1928), ISSN 1469-8064, URL http://dx. doi .org/10.1017/s0305004100011919.

[30] V. Fock, Zeitschrift für Physik A Hadrons and Nuclei 61, 126 (1930), ISSN 1434-6001, URL http: //dx . doi . org/10. $1007 / \mathrm{bf01340294.}$

[31] J. C. Slater, Phys. Rev. 35, 210 (1930), URL http://link. aps.org/doi/10.1103/PhysRev. 35.210.2.

[32] R. Krishnan, J. S. Binkley, R. Seeger, and J. A. Pople, The Journal of Chemical Physics 72 (1980).

[33] H.-J. Werner, P. J. Knowles, G. Knizia, F. R. Manby, M. Schütz, P. Celani, T. Korona, R. Lindh, A. Mitrushenkov, G. Rauhut, et al., Molpro, version 2012.1, a package of ab initio programs (2012), see http://www.molpro.net.

[34] N. A. Zarkevich and D. D. Johnson, The Journal of Chemical Physics 142, 024106+ (2015), ISSN 0021-9606, URL http: //dx.doi.org/10.1063/1.4905209.

[35] G. Henkelman and H. Jónsson, The Journal of Chemical Physics 113 (2000).

[36] E. Bitzek, P. Koskinen, F. Gähler, M. Moseler, and P. Gumbsch, Phys. Rev. Lett. 97, 170201 (2006), URL http://link . aps. org/doi/10.1103/PhysRevLett.97.170201.

[37] J. D. Kemp and K. S. Pitzer, The Journal of Chemical Physics 4, 749 (1936), ISSN 0021-9606, URL http://dx.doi.org/ 10.1063/1.1749784.

[38] S. Klahm and A. Lchow, Chemical Physics Letters 600, 7 (2014), ISSN 0009-2614, URL http: //www.sciencedirect.com/science/article/pii/ S0009261414002048.

[39] M. Baranac-Stojanović, Structural Chemistry pp. 1-8 (2015), ISSN 1040-0400, URL http://dx.doi.org/10.1007/ S11224-014-0557-5.

[40] H. Eyring, D. M. Grant, and H. Hecht, J. Chem. Educ. 39, 466+ (1962), URL http://dx . doi .org/10. 1021/ed039p466.

[41] Nist computational chemistry comparison and benchmark database, nist standard reference database number 101, release 16a, august 2013, editor: Russell d. johnson iii (2013), see http://cccbdb.nist.gov/.

[42] A. V. Kurnosov, A. G. Ogienko, S. V. Goryainov, E. G. Larionov, A. Y. Manakov, A. Y. Lihacheva, E. Y. Aladko, F. V. Zhurko, V. I. Voronin, I. F. Berger, et al., The Journal of Physical Chemistry B 110, 21788 (2006), pMID: 17064141, http://dx.doi.org/10.1021/jp0636726, URL http://dx.doi. org/10.1021/jp0636726.

[43] B. M. Rice and C. F. Chabalowski, J. Phys. Chem. A 5639, 8720 (1997), URL http://pubs.acs.org/doi/abs/10. 
1021/jp972062q.

[44] R. J. Karpowicz and T. B. Brill, J. Phys. Chem. 88, 348 (1984), URL http://pubs.acs.org/doi/abs/10.1021/ j150647a005.

[45] C. S. Choi and E. Prince, Acta Crystallogr., Sect. B: Struct. Sci 28, 2857 (1972), ISSN 05677408, URL http: //scripts . iucr.org/cgi-bin/paper?a09308.

[46] D. I. A. Millar, I. D. H. Oswald, D. J. Francis, W. G. Marshall, C. R. Pulham, and A. S. Cumming, Chem. Commun. 5, 562 (2009), ISSN 1359-7345, URL http://www.ncbi .nlm.nih. gov/pubmed/19283291.

[47] R. Infante-Castillo, L. C. Pacheco-Londoño, and S. P. Hernández-Rivera, J. Mol. Struct. 970, 51 (2010), ISSN 00222860, URL http://linkinghub.elsevier.com/ retrieve/pii/S0022286010001420.

[48] Z. A. Dreger and Y. M. Gupta, J. Phys. Chem. B 10, 3893 (2007), ISSN 1520-6106, URL http://pubs .acs.org/doi/ abs/10.1021/jp0681092.

[49] A. J. Davidson, I. D. H. Oswald, D. J. Francis, A. R. Lennie, W. G. Marshall, D. I. A. Millar, C. R. Pulham, J. E. Warren, and A. S. Cumming, Cryst. Eng. Comm. 10, 162 (2008), ISSN 1466-8033, URL http://pubs.rsc.org/en/ content/articlehtml/2008/ce/b715677b.

[50] J. A. Ciezak, T. A. Jenkins, Z. Liu, and R. J. Hemley, J. Phys. Chem. A 111, 59 (2007).

[51] Z. A. Dreger and Y. M. Gupta, J. Phys. Chem. A 114, 8099 (2010), ISSN 1520-5215, URL http://www.ncbi.nlm.nih. gov/pubmed/20684582.

[52] N. J. Harris and K. Lammertsma, J. Am. Chem. Soc 119, 6583 (1997).
[53] R. W. Molt Jr., T. Watson Jr., V. F. Lotrich, and R. J. Bartlett, J. Phys. Chem. A 115, 884 (2011), ISSN 1520-5215, URL http: //www.ncbi.nlm.nih.gov/pubmed/21210658.

[54] D. I. A. Millar, I. D. H. Oswald, C. Barry, D. J. Francis, W. G. Marshall, C. R. Pulham, and A. S. Cumming, Chemical Communications 46, 5662 (2010), URL http: //dx . doi .org/10. 1039/COCCO0368A.

[55] M. J. Cawkwell, K. J. Ramos, D. E. Hooks, and T. D. Sewell, J. Appl. Phys. 107, 063512 (2010), ISSN 00218979, URL http://link. aip.org/link/JAPIAU/v107/i6/p063512/ s $1 \backslash \&$ Agg=doi.

[56] N. Mathew and R. C. Picu, Chem. Phys. Lett. 582, 78 (2013), ISSN 00092614, URL http://linkinghub. elsevier.com/retrieve/pii/S0009261413009482.

[57] L. B. Munday, R. L. Mitchell, J. Knap, and P. W. Chung, Appl. Phys. Lett. 103, 151911 (2013), ISSN 00036951, URL http://link.aip.org/link/APPLAB/v103/i15/ p151911/s1\\&Agg=doi.

[58] S. M. Avdoshenko and D. E. Makarov, The Journal of Chemical Physics 142, 174106+ (2015), ISSN 0021-9606, URL http: //dx.doi.org/10.1063/1.4919541.

[59] Z. A. Dreger and Y. M. Gupta, J. Phys. Chem. A 114, 7038 (2010).

[60] X. Zheng, J. Zhao, D. Tan, C. Liu, Y. Song, and Y. Yang, Propellants, Explosives, Pyrotechnics 36, 22 (2011), ISSN 1521-4087, URL http://dx. doi .org/10.1002/prep. 200900081.

[61] A. Pereverzev, T. D. Sewell, and D. L. Thompson, The Journal of Chemical Physics 139, 044108+ (2013), ISSN 0021-9606, URL http://dx.doi .org/10.1063/1.4813795. 


\title{
Supplementary Material for:
}

\section{"A Generalized Force-Modified Potential Energy Surface (G-FMPES) for Mechanochemical Simulations"}

\author{
Gopinath Subramanian, ${ }^{1}$ Nithin Mathew ${ }^{2}$ Jeff Leiding ${ }^{3}$ \\ ${ }^{1}$ School of Polymers $\&$ High Performance Materials, \\ University of Southern Mississippi, Hattiesburg, MS, 39402* \\ ${ }^{2}$ Department of Chemistry, University of Missouri-Columbia, Columbia, MO 65211 \\ ${ }^{3}$ Theoretical Division, Los Alamos National Laboratory, Los Alamos, NM 87545
}

\section{Proof that the external force field is conservative}

In order to show that the external force field, $\mathbf{F}_{\text {ext }}(\mathbf{R})$, resulting from our prescription of pseudo-hydrostatic pressure is conservative, we begin with a per-atom description of a system. For a 3-dimensional system containing $N$ atoms, the position vector of the $j^{\text {th }}$ atom, and the external force vector on it are given in component form as

$$
\begin{aligned}
& \mathbf{r}^{(j)}=\left(x^{(j)}, y^{(j)}, z^{(j)}\right) \\
& \mathbf{f}_{\text {ext }}^{(j)}=\left(f_{\text {ext }, x}^{(j)}, f_{\text {ext }, y}^{(j)}, f_{\text {ext }, z}^{(j)}\right)
\end{aligned}
$$

where the various $\left\{\mathbf{f}_{\text {ext }}^{(j)} \forall j=1,2,3 \ldots N\right\}$ make up the external force field $\mathbf{F}_{\text {ext }}(\mathbf{R})$.

The geometric centroid of the configuration is given by the average position of all the atoms in the configuration as

$$
\mathbf{c}=\left(c_{x}, c_{y}, c_{z}\right)=\left(\left\langle x^{(j)}\right\rangle,\left\langle y^{(j)}\right\rangle,\left\langle z^{(j)}\right\rangle\right)
$$

where $\langle\cdot\rangle$ denotes an average taken over all $N$ atoms.

According to our prescription of pseudo-hydrostatic pressure, the external force vector on the $j^{\text {th }}$ atom is given as

$$
\mathbf{f}_{\mathrm{ext}}^{(j)}=P\left[\mathbf{r}^{(j)}-\mathbf{c}\right] \quad ; \quad \forall \quad j=1,2,3 \ldots N
$$

where $P$ is a user-defined "pressure."

The first derivatives of the external force vector (which are the negative elements of the Hessian matrix) are given by

$$
\left\{\frac{\partial \mathbf{f}_{\mathrm{ext}}^{(j)}}{\partial x^{(k)}}, \frac{\partial \mathbf{f}_{\mathrm{ext}}^{(j)}}{\partial y^{(k)}}, \frac{\partial \mathbf{f}_{\mathrm{ext}}^{(j)}}{\partial z^{(k)}} \quad ; \quad \forall \quad j, k=1,2,3 \ldots N\right\}
$$

The first of this set can be written as

$$
\frac{\partial \mathbf{f}_{\mathrm{ext}}^{(j)}}{\partial x^{(k)}}=P\left(\frac{\partial \mathbf{r}^{(j)}}{\partial x^{(k)}}-\frac{\partial \mathbf{c}}{\partial x^{(k)}}\right)
$$

\footnotetext{
* Gopinath.Subramanian@usm.edu; Corresponding author
} 
with

$$
\begin{gathered}
\frac{\partial \mathbf{r}^{(j)}}{\partial x^{(k)}}=\left(\frac{\partial x^{(j)}}{\partial x^{(k)}}, \frac{\partial y^{(j)}}{\partial x^{(k)}}, \frac{\partial z^{(j)}}{\partial x^{(k)}}\right)=\left(\delta_{j k}, 0,0\right) \\
\frac{\partial \mathbf{c}}{\partial x^{(k)}}=\left(\frac{\partial\langle x\rangle}{\partial x^{(k)}}, \frac{\partial\langle y\rangle}{\partial x^{(k)}}, \frac{\partial\langle z\rangle}{\partial x^{(k)}}\right)=\left(\frac{1}{N}, 0,0\right)
\end{gathered}
$$

where $\delta_{j k}$ is the Kronecker delta. Using this result, and similar results for the other derivatives, we obtain the following expressions for all first derivatives of the external force vector as

$$
\begin{aligned}
\frac{\partial \mathbf{f}_{\mathrm{ext}}^{(j)}}{\partial x^{(k)}} & =P\left(\delta_{j k}-\frac{1}{N}, 0,0\right) \\
\frac{\partial \mathbf{f}_{\mathrm{ext}}^{(j)}}{\partial y^{(k)}} & =P\left(0, \delta_{j k}-\frac{1}{N}, 0\right) \\
\frac{\partial \mathbf{f}_{\mathrm{ext}}^{(j)}}{\partial z^{(k)}} & =P\left(0,0, \delta_{j k}-\frac{1}{N}\right)
\end{aligned}
$$

which are all constant for all values of $j$ and $k$ and therefore exist and are continuous everywhere, thereby proving that the external force field is conservative.

\section{The NEB method on a G-FMPES}

With the understanding of how to compute energies, forces, and curvatures on a G-FMPES, the NEB implementation for finding MEPs on a G-FMPES closely follows the original im-

plementation, but with a few crucial modifications. For clarity, our implementation on a G-FMPES is outlined below.

The start and end point structures are re-optimized with the external force and a set of images is initialized between them. Consecutive images are connected by harmonic springs with an equilibrium length of zero and a user-specified spring stiffness $k_{\text {spring }}$ (the actual value of which is not particularly important as long as it is greater than zero, but needs to be on the order of the system forces for efficient convergence). The band is iteratively optimized until the net force on each image is minimized to within a user-specified tolerance. At each iteration, the net force on the $i^{\text {th }}$ image located at $\mathbf{R}^{(i)}$ on the G-FMPES is given by the projected (nudged) forces as:

$$
\overline{\mathbf{F}}_{\text {net,nudged }}^{(i)}=\left[\mathbf{F}_{\text {grad }}^{(i)}+\mathbf{F}_{\text {ext }}^{(i)}\right]_{\perp}+\left[\mathbf{F}_{\text {spring }}^{(i)}\right]_{\|}
$$

where the subscript $\perp$ (or $\|$ ) on a vector indicates its component perpendicular (or parallel) to the unit tangent $\hat{\boldsymbol{\tau}}^{(i)}$. The un-normalized tangent $\boldsymbol{\tau}^{(i)}$ is computed using Henkelman and Jónsson's improved tangent estimate [1]. Defining

$$
\begin{aligned}
& \boldsymbol{\tau}^{(i)+}=\mathbf{R}^{(i+1)}-\mathbf{R}^{(i)} \\
& \boldsymbol{\tau}^{(i)-}=\mathbf{R}^{(i)}-\mathbf{R}^{(i-1)}
\end{aligned}
$$


the tangent is estimated as

$$
\boldsymbol{\tau}^{(i)}=\left\{\begin{array}{lll}
\boldsymbol{\tau}^{(i)+} & \text { if } & \bar{V}^{(i+1)}>\bar{V}^{(i)}>\bar{V}^{(i-1)} \\
\boldsymbol{\tau}^{(i)-} & \text { if } & \bar{V}^{(i+1)}<\bar{V}^{(i)}<\bar{V}^{(i-1)}
\end{array}\right.
$$

where the different $\bar{V}$ are computed using the line integral, as defined in the main article. In the event that the three consecutive values of $\bar{V}$ are neither strictly increasing nor strictly decreasing, i.e. if $\bar{V}^{(i+1)} \leq \bar{V}^{(i)} \geq \bar{V}^{(i-1)}$ or $\bar{V}^{(i+1)} \geq \bar{V}^{(i)} \leq \bar{V}^{(i-1)}$, in order to prevent abrupt switching between two possible tangents, the tangent is taken to be a weighted average as

$$
\boldsymbol{\tau}^{(i)}=\left\{\begin{array}{lll}
\boldsymbol{\tau}^{(i)+} \Delta \bar{V}^{(i)+}+\boldsymbol{\tau}^{(i)-} \Delta \bar{V}^{(i)-} & \text { if } & \bar{V}^{(i+1)}>\bar{V}^{(i-1)} \\
\boldsymbol{\tau}^{(i)+} \Delta \bar{V}^{(i)-}+\boldsymbol{\tau}^{(i)-} \Delta \bar{V}^{(i)+} & \text { if } & \bar{V}^{(i+1)}<\bar{V}^{(i-1)}
\end{array}\right.
$$

with

$$
\begin{aligned}
& \Delta \bar{V}^{(i)+}=\max \left(\left|\bar{V}^{(i+1)}-\bar{V}^{(i)}\right|,\left|\bar{V}^{(i-1)}-\bar{V}^{(i)}\right|\right) \\
& \Delta \bar{V}^{(i)-}=\min \left(\left|\bar{V}^{(i+1)}-\bar{V}^{(i)}\right|,\left|\bar{V}^{(i-1)}-\bar{V}^{(i)}\right|\right)
\end{aligned}
$$

The component of the spring force parallel to the tangent is computed as

$$
\left[\mathbf{F}_{\text {spring }}^{(i)}\right]_{\|}=k_{\text {spring }}\left(\left\|\boldsymbol{\tau}^{(i)+}\right\|-\left\|\boldsymbol{\tau}^{(i)-}\right\|\right) \hat{\boldsymbol{\tau}}^{(i)}
$$

At each iteration, the climbing image method [2] is applied to the highest energy image along the band. This image, identified by $i=h$, is free from all spring forces and is assigned a climbing force computed by inverting the component of the gradient plus external forces along the tangent to this image which is expressed as

$$
\overline{\mathbf{F}}_{\text {net,climb }}^{(i)}=\left(\mathbf{F}_{\text {grad }}^{(i)}+\mathbf{F}_{\text {ext }}^{(i)}\right)-2\left[\left(\mathbf{F}_{\text {grad }}^{(i)}+\mathbf{F}_{\text {ext }}^{(i)}\right) \cdot \hat{\boldsymbol{\tau}}^{(i)}\right] \hat{\boldsymbol{\tau}}^{(i)}
$$

and makes the highest image move uphill along the direction of the first eigenvector, and downhill along all other directions.

In the more recent variation with two climbing images [3], the highest image (with $i=h$ ) is not allowed to climb but is nudged in the usual manner by assigning its net force to be equal to $\overline{\mathbf{F}}_{\text {net,nudged }}$ as in equation 12 . However, its two nearest neighbors (one from each side of the band, i.e., images with $i=h \pm 1$ ) are assigned climbing forces as in equation 20. This prescription results in a higher density of images near the saddle point and is particularly useful for MEPs with unusually high curvatures near the saddle point which would cause the NEB tangent direction to be different from the MEP tangent direction.

Having computed the net force on each image, the band is iteratively optimized until it is well-converged by moving each image towards the point of minimum net force using a suitable optimizer. 


\section{References}

[1] Graeme Henkelman and Hannes Jónsson. Improved tangent estimate in the nudged elastic band method for finding minimum energy paths and saddle points. The Journal of Chemical Physics, 113(22), 2000.

[2] Graeme Henkelman, Blas P. Uberuaga, and Hannes Jónsson. A climbing image nudged elastic band method for finding saddle points and minimum energy paths. The Journal of Chemical Physics, 113(22), 2000.

[3] Nikolai A. Zarkevich and Duane D. Johnson. Nudged-elastic band method with two climbing images: Finding transition states in complex energy landscapes a). The Journal of Chemical Physics, 142(2):024106+, January 2015. 